\section{Anticoagulação na fase aguda}

A HNF é efetiva no tratamento de TEP, mostrando melhores resultados quando comparada à condição de não tratamento do paciente com TEP confirmada. ${ }^{(1)}$ Devem ser respeitadas as contraindicações ao seu uso, pesando a relação risco-benefício de acordo com a situação clínica do paciente (Quadro 10). ${ }^{(2,3)}$ A dose da HNF calculada pelo peso do paciente - a dose de ataque de $80 \mathrm{UI} / \mathrm{kg}$ e a dose de manutenção de $18 \mathrm{Ul} / \mathrm{kg}$ i.v., ajustada a cada $6 \mathrm{~h}$ de acordo com o valor de TTPa medido - resulta em um tempo menor para se atingir os valores alvos de TTPa entre 1,5 e 2,3 vezes o controle, quando comparada a doses padronizadas de ataque de $5.000 \mathrm{Ul}$ e a doses de manutenção de $1.000 \mathrm{Ul} / \mathrm{h} .{ }^{(4)}$ No Quadro 11 está disposto um nomograma para uso da HNF i.v. de forma contínua.

Recentemente, estudos mostraram que a HBPM s.c., com dose calculada de acordo com o peso do paciente, é pelo menos tão eficaz quanto a HNF i.v. com dose ajustada de acordo com os controles periódicos de TTPa. Em duas meta-análises, verificou-se maior facilidade de administração da HBPM, assim como houve resultados favoráveis a ela em relação à HNF quanto à mortalidade e à ocorrência de sangramentos graves, fatos esses que permitem a sua indicação como primeira escolha no tratamento de TEP não maciça. ${ }^{(5-13)}$ Em um recente ensaio clínico randomizado no Canadá, comparouse o uso de HNF s.c. em dose fixa por $\mathrm{kg}$ de peso (333 Ul $/ \mathrm{kg}$ seguido por $250 \mathrm{Ul} / \mathrm{kg}$ a cada 12 h), sem monitorização de TTPa, com HBPM, não havendo redução da eficácia ou aumento de efeitos adversos. Esse regime pode ser uma alternativa terapêutica.(B) ${ }^{(10)}$

A administração da HBPM pode ser feita em duas aplicações diárias ou em apenas uma, sem prejuízo do efeito anticoagulante ou aumento do risco de sangramentos documentados até o momento. ${ }^{(10-13)}$ Não existem estudos consistentes demonstrando diferenças das diversas HBPM no tratamento da TEP. Em pacientes com função renal normal, a enoxaparina é utilizada na dose de $1 \mathrm{mg} / \mathrm{kg}$ de peso a cada $12 \mathrm{~h}$ ou $1,5 \mathrm{mg} / \mathrm{kg}$ a cada $24 \mathrm{~h}$, a nadroparina é utilizada na dose de $90 \mathrm{Ul} / \mathrm{kg}$ a cada $12 \mathrm{~h}$ ou $190 \mathrm{Ul} / \mathrm{kg}$ a cada $24 \mathrm{~h}$, e a dalteparina é utilizada na dose de $120 \mathrm{Ul} / \mathrm{kg}$ a cada $12 \mathrm{~h}$ ou $200 \mathrm{Ul} / \mathrm{kg}$ a cada $24 \mathrm{~h}$.

Pacientes com diagnóstico objetivo de TEP aguda não maciça devem ser tratados com
HNF ou com HBPM,(A) dando-se preferência à HBPM.(A)

Por se tratar de doença grave, com grande potencial de morbidade e mortalidade, não é necessária a confirmação diagnóstica para o início do tratamento, em caso de forte suspeita clínica. No entanto, o tratamento definitivo com anticoagulantes orais só deverá ser iniciado após a confirmação diagnóstica, tendo em vista os riscos envolvidos com o uso dessas medicações. ${ }^{(14,15)}$

Embora não haja evidência na literatura, o início da ação da HBPM é mais lento que a da HNF i.v. Por esse motivo, pode-se fazer uma dose de ataque com HNF i.v. de $5.000 \mathrm{Ul}$ associada à dose inicial de HBPM. ${ }^{(14)}$

Para pacientes com suspeita clínica forte de TEP, aguardando definição diagnóstica, recomenda-se o início da anticoagulação com HNF ou HBPM. Deve-se fazer a dose inicial da HBPM, associada a uma dose de ataque de $5.000 \mathrm{UI}$ de HNF.(D)

Trabalhos clínicos não randomizados mostraram uma maior chance de sangramentos importantes relacionados ao uso de HBPM em pacientes com insuficiência renal grave, uma vez que a excreção dessa droga é feita por via renal. Se, ainda assim, a opção for pelo uso de HBPM, deve-se fazer o controle com a medida do antifator Xa. Em pacientes obesos, ainda não há dados que justifiquem a contraindicação de HBPM. Os trabalhos que avaliaram esse subgrupo usaram o peso atual dos pacientes para o cálculo da dose da HBPM a ser usada. ${ }^{(16-19)}$

Em pacientes com insuficiência renal grave, deve-se usar preferencialmente a HNF ao invés da HBPM.(C)

A infusão contínua de HNF i.v. mostrouse superior à administração intermitente s.c. quanto à recorrência de eventos trombóticos e à maior chance de sangramentos. ${ }^{(20-21)}$ Portanto, sempre que possivel, deve-se optar pela infusão contínua i.v. para a infusão de heparina.

A HNF deve ser usada preferencialmente em regime de infusão contínua i.v.(B)

A combinação entre reagentes e coagulômetros pode fornecer valores muito irregulares de TTPa, resultando na inadequação da anticoagulação (excessiva ou insuficiente). Para evitar esse tipo de erro, sugere-se a dosagem de heparina plasmática e que o intervalo de TTPa corresponda à dosagem plasmática de $0,3-0,7 \mathrm{Ul} / \mathrm{mL}$ 
Quadro 10 - Contraindicações relativas para o uso de anticoagulantes.

\begin{tabular}{l}
\hline Sangramento ativo ${ }^{\text {a }}$ \\
Doença ulcerosa péptica ativa \\
Defeitos conhecidos na coagulação \\
(RNI basal > 1,2 ou TTPa $>1,3$ vs. controle) \\
Trombocitopenia $\left(<50.000 / \mathrm{mm}^{3}\right.$ ) ou disfunção \\
plaquetária \\
AVC hemorrágico recente ${ }^{a}$ \\
Paciente com dificuldade de adesão ao tratamento \\
(por motivos clínicos e/ou sociais) \\
História de quedas (3 dentro do último ano ou \\
recorrente lesão por queda) \\
Hipertensão arterial sistêmica não controlada \\
(> 180/110 mmHg) \\
Cirurgia maior ou politraumatismo nos últimos \\
3 meses \\
Cirurgia maior ou procedimento invasivo planejado \\
Endocardite bacteriana \\
Doença intracerebral ativa (por ex., metástase cerebral \\
confirmada) \\
Anemia grave ou de causa não explicada \\
Tumores ulcerados (de qualquer tipo) \\
Gestação ${ }^{\text {b }}$ \\
TlH
\end{tabular}

${ }^{a}$ Recomenda-se aguardar pelo menos 4-6 semanas para reiniciar a anticoagulação em hemorragias fora do SNC. Em hemorragia intracraniana, deve-se esperar mais tempo. Caso haja opção não farmacológica eficaz, essa deve ser instituída neste período (p.ex., uso de filtro de veia cava em paciente com TEP grave e recorrente). 'bestação é contraindicação somente em relação aos AVK. 'Contraindicação somente ao uso de HNF ou HBPM. Adaptado de estudos anteriores. ${ }^{(2,3)}$

(para o lote de reagente testado). No entanto, se o laboratório não tem as condições de dosar a heparina, deve-se adotar o intervalo de TTPa entre 1,5 e 2,3 vezes o valor de referência do paciente, pois esse intervalo é geralmente mais adequado para os reagentes modernos. ${ }^{(22,23)}$

Em pacientes tratados com HNF, o valor de controle de TTPa deve corresponder à dosagem de heparina plasmática de 0,3-0,7 UI/mL, de acordo com testes que avaliem a inibição do fator Xa. Entretanto, na impossibilidade da dosagem da heparina sérica pelo laboratório e subsequente estabelecimento do intervalo adequado de TTPa a ser seguido, deve-se adotar o intervalo entre 2,5 e 3,5 vezes o valor de base do paciente.(B)

Alguns pacientes apresentam resistência à heparina, definida como a necessidade diária de HNF (infusão contínua i.v.) maior que $40.000 \mathrm{UI} /$ dia. Nessa situação, o controle feito pela medida do antifator Xa diminui a necessidade de aumentos adicionais da dose da HNF. (24)

Pacientes em tratamento com infusão contínua de HNF i.v que estejam necessitando de doses excessivamente altas (maiores que $40.000 \mathrm{Ul} / \mathrm{dia}$ ), para atingir os alvos propostos de TTPa, devem passar a ser controlados com testes para dosagem de antifator Xa.(B)

0 fondaparinux sódico é um anticoagulante inibidor direto do fator Xa utilizado tanto no tratamento como na profilaxia de TEV. ${ }^{(15)}$ Possui registro na ANVISA, mas ainda não é comercializado no Brasil. Em um grande ensaio clínico aberto de não inferioridade, comparou-se o fondaparinux com a HNF por infusão contínua i.v., não demonstrando diferenças em relação à recorrência de TEV e sangramento. ${ }^{(25)}$ Como não há descrição de $\mathrm{TIH}$, não há necessidade de se monitorar a contagem das plaquetas. Pela sua longa meia-vida (15-20 h), o medicamento pode ser administrado uma vez ao dia. A dose varia conforme o peso corporal, sendo de $5 \mathrm{mg} / \mathrm{dia}$ para pacientes com peso abaixo de $50 \mathrm{~kg}$, de $7,5 \mathrm{mg} / \mathrm{dia}$ se o peso for de $50-100 \mathrm{~kg}$ e de $10 \mathrm{mg} /$ dia para aqueles com peso maior que $100 \mathrm{~kg}$. É contraindicado em pacientes com insuficiência renal grave (depuração de creatinina endógena $<20 \mathrm{~mL} / \mathrm{min}) .{ }^{(26)}$

A TIH é uma complicação grave relacionada à terapia com heparina. A PIH tipo 1 é uma forma menos grave, provavelmente relacionada ao efeito direto da heparina na ativação plaquetária e sem mediação imunológica. A forma imunomediada, também chamada de PIH tipo 11, é causada por imunoglobulinas direcionadas contra o fator 4 do complexo heparina. ${ }^{(27)} \mathrm{A}$ incidência de PIH tipo 11 varia de 1-3\%. Um dos fatores que parece influenciar a ocorrência dessa complicação é o tipo de heparina (HNF > HBPM $>$ fondaparinux); porém, em uma meta-análise sobre esse assunto, não se confirmou a menor incidência dessa complicação com o uso da HBPM quando comparado com o uso de HNF. (28) Geralmente a PlH ocorre após 5-14 dias do início do tratamento ou mais precocemente em casos de reexposição. Caracteriza-se por plaquetopenia (plaquetas inferiores a $100.000 / \mathrm{mm}^{3}$ ou em número inferior a 50\% do valor basal do paciente) e aumento de risco de eventos trombóticos venosos e arteriais. Define-se o diagnóstico 
Quadro 11 - Nomograma para o uso de HNF.

\begin{tabular}{|ll|}
\hline Dose de ataque & $80 \mathrm{Ul} / \mathrm{kg}$ i.v. em bolus \\
Dose inicial da infusão contínua & \\
Ajuste da infusão por TTPa & \\
$\quad$ Valor medido em TTPa & $18 \mathrm{Ul} / \mathrm{kg}$ a cada h (i.v.) \\
$<1,2 \times$ controle & Ajuste \\
$1,2-1,5 \times$ controle & $80 \mathrm{Ul} / \mathrm{kg}$ i.v. em bolus + aumentar infusão em $4 \mathrm{Ul} / \mathrm{kg}$ a cada h \\
$1,6-2.3 \times$ controle & $40 \mathrm{Ul} / \mathrm{kg}$ i.v. em bolus + aumentar infusão em $2 \mathrm{Ul} / \mathrm{kg}$ a cada h \\
$2,4-3,0 \times$ controle & Não modificar \\
$>3,0 \times$ controle & Diminuir a infusão em $2 \mathrm{Ul} / \mathrm{kg}$ a cada h \\
\hline
\end{tabular}

a Sugestão de preparo da solução de HNF: soro glicosado 5\% ou soro fisiológico 0.9\% (99 mL) e HNF 5.000 Ul (1 mL). Concentração final: $50 \mathrm{Ul} / \mathrm{mL}$. 'Após cada ajuste na dose de HNF, deve-se solicitar uma nova dosagem de TTPa em 6 h. Ficando o nível estável, repete-se o TTPa a cada dia durante o uso de HNF. Adaptado do estudo de Raschke et al. ${ }^{(4)}$

por exclusão de outras causas de plaquetopenia e através de testes imunológicos específicos. ${ }^{(27)}$ 0 tratamento consiste em suspender a heparina e iniciar a medicação anticoagulante alternativa. Entre as recomendadas, estão os inibidores diretos da trombina, lepirudina e argatrobana. ${ }^{(29)}$ 0 uso de AVK geralmente é contraindicado na fase aguda de doença.

Os anticoagulantes orais atuam inibindo os fatores da coagulação dependentes da vitamina K (fatores Il, VII, IX e X), mas eles também reduzem a sintese de fatores anticoagulantes naturais, proteínas $\mathrm{C}$ e $\mathrm{S}$, o que pode promover eventos trombóticos paradoxais no início do tratamento, enquanto a ação anticoagulante não for plena. Antigamente, o anticoagulante oral só era iniciado após cerca de 5 dias de uso contínuo de HNF; entretanto, estudos prospectivos randomizados mostraram que não havia diferença quanto à recorrência de eventos trombóticos entre o início precoce (menos de 3 dias após o início da anticoagulação com heparina) e o início tardio (após 5 dias). Por outro lado, o início precoce, concomitante ao início da heparina, diminui o tempo de internação hospitalar, sendo, portanto, recomendado. A dose inicial deve ser de varfarina 5-10 mg/dia nos primeiros 3 dias, seguindo-se o ajuste de acordo com a RNI. ${ }^{(30-33)}$ Em nosso meio, além da varfarina, há a femprocumona. Entretanto, esse anticoagulante oral, que tem meia-vida mais longa, é menos estudado e não há nomogramas específicos de uso, sendo, portanto, dada a preferência à varfarina.

0 anticoagulante oral só deve ser começado após a confirmação diagnóstica de TEP. Deve ser iniciado em conjunto com HNF ou HBPM, que poderá ser suspensa tão logo o controle da anti- coagulação oral tenha atingido o alvo (RN1 entre 2 e 3 , por pelo menos $24 \mathrm{~h}$ ) e tempo de sobreposição entre heparinas e AVK de no mínimo 5 dias.(A)

\section{Referências}

1. Barrit DW, Jordan SC. Anticoagulant drugs in the treatment of pulmonary embolism. A controlled trial. Lancet. 1960;1 (7138):1309-12.

2. Institute for Clinical System Improvement. Anticoagulant Therapy Supplement. Bloomington: Institute for Clinical System Improvement; 2001.

3. Samama MM, Cohen AT, Darmon JY, Desjardins L, Eldor A, Janbon C, et al. A comparison of enoxaparin with placebo for the prevention of venous thromboembolism in acutely ill medical patients. Prophylaxis in Medical Patients with Enoxaparin Study Group. N Engl J Med. 1999;341(11):793-800.

4. Raschke RA, Reilly BM, Guidry JR, Fontana JR, Srinivas S. The weight-based heparin dosing nomogram compared with a "standard care" nomogram. A randomized controlled trial. Ann Intern Med. 1993;119(9):874-81.

5. de Valk HW, Banga JD, Wester JW, Brouwer CB, van Hessen MW, Meuwissen OJ, et al. Comparing subcutaneous danaparoid with intravenous unfractionated heparin for the treatment of venous thromboembolism. A randomized controlled trial. Ann Intern Med. 1995;123(1):1-9.

6. Simonneau G, Sors H, Charbonnier B, Page Y, Laaban JP, Azarian R, et al. A comparison of low-molecular-weight heparin with unfractionated heparin for acute pulmonary embolism. The THESEE Study Group. Tinzaparine ou Heparine Standard: Evaluations dans l'Embolie Pulmonaire. N Engl J Med. 1997;337(10):663-9.

7. van Dongen $\mathrm{CJ}$, van den Belt $\mathrm{AG}$, Prins $\mathrm{MH}$, Lensing AW. Fixed dose subcutaneous low molecular weight heparins versus adjusted dose unfractionated heparin for venous thromboembolism. Cochrane Database Syst Rev. 2000;(2):CD001100.

8. Hull RD, Raskob GE, Brant RF, Pineo GF, Elliott G, Stein $\mathrm{PD}$, et al. Low-molecular-weight heparin vs heparin in the treatment of patients with pulmonary embolism. American-Canadian Thrombosis Study Group. Arch Intern Med. 2000;160(2):229-36. 
9. Dolovich LR, Ginsberg JS, Douketis JD, Holbrook AM, Cheah G. A meta-analysis comparing low-molecularweight heparins with unfractionated heparin in the treatment of venous thromboembolism: examining some unanswered questions regarding location of treatment, product type, and dosing frequency. Arch Intern Med. 2000;160(2):181-8.

10. Couturaud F, Julian JA, Kearon C. Low molecular weight heparin administered once versus twice daily in patients with venous thromboembolism: a meta-analysis. Thromb Haemost. 2001;86(4):980-4.

11. van Dongen $\mathrm{CJ}$, MacGillavry MR, Prins MH. Once versus twice daily $\mathrm{LMWH}$ for the initial treatment of venous thromboembolism. Cochrane Database Syst Rev. 2005;(3):CD003074.

12. Kearon C, Ginsberg JS, Julian JA, Douketis J, Solymoss S, Ockelford P, et al. Comparison of fixeddose weight-adjusted unfractionated heparin and low-molecular-weight heparin for acute treatment of venous thromboembolism. JAMA. 2006;296(8):935-42.

13. Merli G, Spiro TE, Olsson CG, Abildgaard U, Davidson BL, Eldor A, et al. Subcutaneous enoxaparin once or twice daily compared with intravenous unfractionated heparin for treatment of venous thromboembolic disease. Ann Intern Med. 2001;134(3):191-202.

14. British Thoracic Society Standards of Care Committee Pulmonary Embolism Guideline Development Group. British Thoracic Society guidelines for the management of suspected acute pulmonary embolism. Thorax. 2003;58(6):470-83.

15. Kearon C, Kahn SR, Agnelli G, Goldhaber S, Raskob GE, Comerota AJ, et al. Antithrombotic therapy for venous thromboembolic disease: American College of Chest Physicians Evidence-Based Clinical Practice Guidelines (8th Edition). Chest. 2008;133(6 Suppl):454S-545S.

16. Gerlach AT, Pickworth KK, Seth SK, Tanna SB, Barnes JF. Enoxaparin and bleeding complications: a review in patients with and without renal insufficiency. Pharmacotherapy. 2000;20(7):771-5.

17. Cestac P, Bagheri H, Lapeyre-Mestre M, Sié P, Fouladi A, Maupas E, et al. Utilisation and safety of low molecular weight heparins: prospective observational study in medical inpatients. Drug Saf. 2003;26(3):197-207.

18. Spinler SA, Inverso SM, Cohen M, Goodman SG, Stringer KA, Antman EM, et al. Safety and efficacy of unfractionated heparin versus enoxaparin in patients who are obese and patients with severe renal impairment: analysis from the ESSENCE and TIMI 11B studies. Am Heart J. 2003;146(1):33-41.

19. Wilson SJ, Wilbur K, Burton E, Anderson DR. Effect of patient weight on the anticoagulant response to adjusted therapeutic dosage of low-molecular- weight heparin for the treatment of venous thromboembolism. Haemostasis. 2001;31(1):42-8.

20. Hull RD, Raskob GE, Hirsh J, Jay RM, Leclerc JR, Geerts $\mathrm{WH}$, et al. Continuous intravenous heparin compared with intermittent subcutaneous heparin in the initial treatment of proximal-vein thrombosis. N Engl J Med. 1986;315(18):1109-14.
21. Glazier RL, Crowell EB. Randomized prospective trial of continuous vs intermittent heparin therapy. JAMA. 1976;236(12):1365-7.

22. Bates SM, Weitz Jl, Johnston M, Hirsh J, Ginsberg JS. Use of a fixed activated partial thromboplastin time ratio to establish a therapeutic range for unfractionated heparin. Arch Intern Med. 2001;161(3):385-91.

23. Hirsh J, Raschke R. Heparin and low-molecularweight heparin: the Seventh ACCP Conference on Antithrombotic and Thrombolytic Therapy. Chest. 2004;126(3 Suppl):188S-203S.

24. Torbicki A, Perrier A, Konstantinides S, Agnelli G, Galiè $N$, Pruszczyk P, et al. Guidelines on the diagnosis and management of acute pulmonary embolism: the Task Force for the Diagnosis and Management of Acute Pulmonary Embolism of the European Society of Cardiology (ESC). Eur Heart J. 2008;29(18):2276-315.

25. Büller HR, Davidson BL, Decousus H, Gallus A, Gent M, Piovella F, et al. Subcutaneous fondaparinux versus intravenous unfractionated heparin in the initial treatment of pulmonary embolism. $\mathrm{N}$ Engl J Med. 2003;349(18):1695-702. Erratum in: N Engl J Med. 2004;350(4):423.

26. Levine MN, Hirsh J, Gent M, Turpie AG, Cruickshank M, Weitz $\mathrm{J}$, et al. A randomized trial comparing activated thromboplastin time with heparin assay in patients with acute venous thromboembolism requiring large daily doses of heparin. Arch Intern Med. 1994;154(1):49-56.

27. Warkentin TE. Heparin-induced thrombocytopenia: diagnosis and management. Circulation. 2004;110(18):e454-8.

28. Morris TA, Castrejon S, Devendra G, Gamst AC. No difference in risk for thrombocytopenia during treatment of pulmonary embolism and deep venous thrombosis with either low-molecular-weight heparin or unfractionated heparin: a metaanalysis. Chest. 2007;132(4):1131-9.

29. Greinacher A, Völpel H, Janssens U, Hach-Wunderle V, Kemkes-Matthes B, Eichler P, et al. Recombinant hirudin (lepirudin) provides safe and effective anticoagulation in patients with heparin-induced thrombocytopenia: a prospective study. Circulation. 1999;99(1):73-80.

30. Gallus A, Jackaman J, Tillett J, Mills W, Wycherley A. Safety and efficacy of warfarin started early after submassive venous thrombosis or pulmonary embolism. Lancet. 1986;2(8519):1293-6.

31. Rosiello RA, Chan CK, Tencza F, Matthay RA. Timing of oral anticoagulation therapy in the treatment of angiographically proven acute pulmonary embolism. Arch Intern Med. 198;147(8):1469-73.

32. Hull RD, Raskob GE, Rosenbloom D, Panju AA, BrillEdwards P, Ginsberg JS, et al. Heparin for 5 days as compared with 10 days in the initial treatment of proximal venous thrombosis. $N$ Engl $\mathrm{J}$ Med. 1990;322(18):1260-4.

33. Institute for Clinical Systems Improvement. Venous Thromboembolism Diagnosis and Treatment. Bloomington: Institute for Clinical System Improvement; 2009. 